\title{
Longer characteristic wavelength in a novel engineered photoprotein Mnemiopsin 2
}

\author{
Mahsa Hematyar ${ }^{1} \cdot$ Vahab Jafarian $^{1} \cdot$ Akram Shirdel $^{2}{ }^{\oplus}$
}

Received: 12 November 2021 / Accepted: 14 February 2022 / Published online: 28 February 2022

(c) The Author(s), under exclusive licence to European Photochemistry Association, European Society for Photobiology 2022

\begin{abstract}
We designed two mutants of photoprotein Mnemiopsin 2 (Mn2) including M52I and V144I, where the mutations were applied in the EF-hand loops I and III. Far-UV CD measurements demonstrated that the stability of the helices in the wild-type (WT) protein is greater compared with the mutants. Heat-induced denaturation experiments in the apo-form of photoproteins showed that WT Mn2 has higher value of the enthalpy change for the unfolding process, indicating that it has more stabilizing interaction compared with mutants. According to the activity measurement data, both mutants, particularly V144I have lower initial intensity as well as slower decay rate as compared with the WT photoprotein. Importantly, it was found that V144I variant shows $25 \mathrm{~nm}$ of red shift in the characteristic wavelengths as compared with the WT photoprotein. This finding can be considered as an advantage for in vivo application of photoprotein for imaging purposes. It concluded that this position on loop III of $\mathrm{Mn} 2$ is a hotspot point for characteristic wavelength determination. However, further research on this mutant is needed for making stable variants of Mn2 with novel optical features.
\end{abstract}

Keywords Photoprotein $\cdot$ Mnemiopsin $2 \cdot$ Mutation $\cdot$ Initial intensity $\cdot$ Decay rate $\cdot$ Optical feature

\section{Introduction}

Bioluminescence is an interesting phenomenon of light emission in biological systems. This appealing phenomenon has found in a broad range of marine and terrestrial animals. Up to now, more than 30 bioluminescence systems were genetically and biochemically characterized $[1,2]$. Bioluminescence involves two distinctive mechanisms. The first one uses a class of oxidative enzymes known as luciferase that catalyzes the oxidation of luciferin to into unstable oxyluciferin that emit light to obtain its stable form in the ground state.

The $\mathrm{Ca}^{+2}$-regulated photoproteins are unique members of bioluminescence systems that uses the second mechanism of light emission in living organisms [3, 4]. It involves the

Vahab Jafarian

V.Jafarian@znu.ac.ir

$\triangle$ Akram Shirdel

a.shirdel@modares.ac.ir

1 Department of Biology, Faculty of Sciences, University of Zanjan, Zanjan, Iran

2 Department of Biochemistry, Faculty of Biological Sciences, Tarbiat Modares University, Tehran, Iran formation of photoprotein-substrate complex by the noncovalent attachment of 2-hydroperoxycoelenterazine to the apoprotein. Upon coordination of $\mathrm{Ca}^{+2}$ into EF-hand loops, the tertiary structure of photoprotein undergoes conformational alternation followed by the production of coelenteramide in the excited state form. Therefore, the electronically excited coelenteramide relaxes to its corresponding ground state that is accompanied by the emission of flash-light in the visible region [5].

Depending on the type of photoprotein, the emission spectra of photoproteins ranges from 465 to $495 \mathrm{~nm}$ corresponding to the blue region of the electromagnetic spectrum [6]. It has proposed that special interactions including H-bonds, $\pi-\pi$, and hydrophobic one between the core structure of the photoprotein and coelenterazine as well as the electronic conjugation of the aromatic rings of coelenterazine have critical roles in determining the optical features of the emitted light. These weak interactions play fundamental roles in determining the maximum characteristic wavelength of the light [7]. It is worthwhile to mention that $\mathrm{Ca}^{+2}$ speeds up bioluminescence reaction process, and it proceeds slowly in the absence of $\mathrm{Ca}^{+}[8]$.

It has revealed that $\mathrm{Ca}^{+2}$-regulated photoprotein are made up of a single polypeptide chain, and form a compact 
and globular structure [9]. According to X-ray crystallography studies, photoproteins generally consist of apophotoprotein that are complexed with their substrate to form functional complexes. Besides, in apophotoprotein form, a photoprotein contains four helix-loop-helix motifs (known as EF-hand motifs) and a hydrophobic cavity located in the core structure of protein for substrate binding $[10,11]$. EF-hand motifs provide particular sequence for $\mathrm{Ca}^{+2}$ coordination [12]. In other words, twelve conserved amino acid residues were characterized in the loop region in which residues $1,3,5,9$, and 12 supply oxygen atoms to interact with the $\mathrm{Ca}^{+2}$. The variable amino acids occurred in positions $2,4,10,11$, while position 6 is occupied by Glycine [13, 14]. A remarkable study by Tricoire and his colleagues has been revealed that all of three EF-hands of aequorin including I/III/IV act as functional units for binding to $\mathrm{Ca}^{+2}$ that is essential for triggering of bioluminescence [15]. Additionally, EF-hands I/III rather than EF-hand IV have more affinity to $\mathrm{Ca}^{+2}$ ions. It was shown that EF-hand loop II of cnidarians and ctenophore families have not the canonical sequence for binding to $\mathrm{Ca}^{2+}[16,17]$.

Considering the significant advantages of bioluminescent proteins such as better penetration depth, higher signal-tonoise ratio, superior detection sensitivity and non-invasive character, they become valuable biochemical tools with a wide ranges of applications in bio-imaging, biosensing, reporters in immunoassays, gene expression analysis, environmental monitoring, bio-threat evaluation and hybridization assays [16-20]. Therefore, according to the area of application, different bioluminescence emission wavelengths of photoproteins can be used to develop and optimize their applications.

Different techniques, such as random and site-directed mutagenesis have been used to make a variety of photoprotein with altered bioluminescent properties including initial intensity, decay rate, characteristic emission wavelengths, and sensitivity to $\mathrm{Ca}^{+2}$ ions $[16,20,21]$.

Mnemiopsin $2(\mathrm{Mn} 2)$ is a member of photoproteins, which is produced naturally by the photocyte cell of Mnemiopsis leidyi. It has only one polypeptide chain composed of 207 amino acid residues weighing around $24 \mathrm{kDa}[22,23]$. In previous work on loops III and IV of Mn2, we proposed a model in which simultaneous occurrence of Gly in loops III and IV is essential for evolutionary adjustment of initial intensity and decay rate of luminescence emission [24]. In this study, we aimed to shed a light on the role of representative residues in the first and third loop of Mn2 on the structure and activity of photoprotein using a combination of the site-directed mutagenesis and spectroscopic methods. Interestingly, it was found that a point mutation on the loop III can shift the characteristic maximum emission toward longer wavelength.

\section{Materials and methods}

\subsection{Materials}

DpnI and Pfu were purchased from Thermo Scientific (Vilnius, Lithuania). The cp-coelenterazine from Sigma (St. Louis, MO, USA). Kanamycin and IPTG (Isopropyl $\beta$-D-1-thiogalactopyranoside) and plasmid extraction kit were obtained from Invitrogen (Carlsbad, CA, USA), Qiagen (Hilden, Germany) and Bioneer (Seoul, South Korea), respectively. Other chemicals were prepared from Merck (Merck, Darmstadt, Germany).

\subsection{Bioinformatics studies}

Similarity search between the sequence of $\mathrm{Mn} 2$ and nonredundant protein sequences was performed by the BLAST program under the NCBI database [25]. Local pairwise sequence alignment between the sequence of $\mathrm{Mn} 2$ and representative similar sequences was done by the Emboss matcher program [26]. Multiple sequence alignment was performed by the CLUSTALW program [27] and the output of the CLUSTALW was saved and used as input for the ESPript program [28].

Three-dimensional structural model of the WT Mn2 and its mutants were made by the MODELLER program, Ver 10.1 [29] using the crystal structure of berovin (PDB ID: 5BPJ) as the template [10] (90\% identity) and crystal structure of aequorin (PDB ID: 1EJ3) [30] for docking of coelenterazine into the cavity of photoprotein. The quality of structural models was evaluated by comparing the Z-Dope score of the structures by the MODELLER program [31]. Finally, the best structure for individual variants was selected depending on the quality score of the programs. Graphical representation of the structures was done by UCSF CHIMERA program Ver. 1.13.1 [32].

\subsection{Protein engineering}

Plasmid carrying the gene of apo-Mn2 (GenBank accession No. GQ884175), previously has cloned into pET-28a [33]. Its amplification was performed by site-directed mutagenesis method. Two mutagenic primers for each mutation were synthesized, and PCR amplification of DNA was done by denaturation at $94{ }^{\circ} \mathrm{C}$ for $3 \mathrm{~min}, 23$ cycles of $94{ }^{\circ} \mathrm{C}$ for $1 \mathrm{~min}, 52{ }^{\circ} \mathrm{C}$ (V144I mutant) and $57^{\circ} \mathrm{C}$ (M52I mutant) for $1 \mathrm{~min}, 68^{\circ} \mathrm{C}$ for $12 \mathrm{~min}$ and a final extension temperature at $68{ }^{\circ} \mathrm{C}$ for $10 \mathrm{~min}$. Digestion of the native parental plasmids was done by incubation of the PCR products with DpnI at $37{ }^{\circ} \mathrm{C}$ for $20 \mathrm{~h}$. Then the reaction product was transformed into the Escherichia coli 
DH5a cells. Finally, the existence of the mutations was verified by DNA sequencing.

\subsection{Expression and purification}

To construct the expression system for the WT and mutant photoproteins, the plasmids containing the WT sequence and designed mutations were transformed into Escherichia coli BL21 (DE3) using chemical methods. The cells were cultured at $37{ }^{\circ} \mathrm{C}$ in $200 \mathrm{ml}$ Terrific broth (TB) medium containing $35 \mu \mathrm{g} / \mathrm{ml}$ Kanamycin and were allowed growing to reach the optical density of $0.6-0.8(620 \mathrm{~nm})$. IPTG $(1 \mathrm{mM})$ was added to the TB solution and cells were permitted to consume it at $28{ }^{\circ} \mathrm{C}$ for $6 \mathrm{~h}$. At the end of expression, cells deposits were gathered by centrifugation at $3500 \mathrm{~g}$ for $20 \mathrm{~min}$ and $4{ }^{\circ} \mathrm{C}$. Purification of recombinant apophotoproteins was done by $\mathrm{Ni}-\mathrm{NTA}$ resin. The eluted samples were collected for dialysis, and $12.5 \%$ SDS-PAGE analysis. Protein concentration was determined by the Bradford method [34].

\subsection{Photoprotein characterization and bioluminescent activity measurements}

To make preparations of semi-synthetic $\mathrm{Mn} 2$, the dialyzed apomnemiopsins were incubated in a dark environment at $4{ }^{\circ} \mathrm{C}$ with cp-coelenterazine ( $8 \mu \mathrm{M}$ concentration in final volume) in $50 \mathrm{mM}$ Tris-based buffer (pH 9.0) containing $10 \mathrm{mM}$ EDTA (buffer 1 ) at $4{ }^{\circ} \mathrm{C}$. After $15 \mathrm{~h}, 5 \mu \mathrm{l}$ of the incubate solution with a $45 \mu$ of buffer 2 (50 mM Tris-base, $\mathrm{pH}$ 9.0) were mixed in a glass tube. The luminescence activity was measured by injection of $100 \mu \mathrm{l}$ Tris-base $(50 \mathrm{mM})$ containing $40 \mathrm{mM} \mathrm{CaCl}_{2}, \mathrm{pH}$ 9.0 (buffer 3) into the sample solution in a luminometer (Sirius tube luminometer, Berthold Detection System, Germany. The sensitivity to calcium ion was done based on the method proposed by Inouye [35, 36].

\subsection{Determination of bioluminescence emission spectra}

Bioluminescence emission spectra were recorded in the wavelength range between 400 and $700 \mathrm{~nm}$ with a Cytation 5 (Biotek, USA) using a 96-well microtiter plate. $50 \mu \mathrm{l}$ of incubated protein samples at $50 \mathrm{mM}$ Tris-base buffer containing $40 \mathrm{mM} \mathrm{CaCl}_{2}, \mathrm{pH} 9.0$ were put in wells (at room temperature, where the excitation light source turned off. All photoprotein spectra were measured in triplicate, blank corrected.

\subsection{Spectroscopy}

\subsubsection{Circular dichroism (CD)}

Circular dichroism (CD) spectra at the far-UV region $(185-250 \mathrm{~nm})$ were recorded with a Circular Dichroism
Spectropolarimeter (Model-215 Aviv company, USA) using a cuvette with a path-length of $1 \mathrm{~mm}$. The concentration of $0.2 \mathrm{mg} \mathrm{ml}^{-1}$ at $\mathrm{pH} 9.0$ was used for the spectral measurements. All measurements were done at and $25^{\circ} \mathrm{C}$.

\subsection{Intrinsic and extrinsic Fluorescence measurements}

The intrinsic and ANS-based fluorescence spectra were recorded for evaluation of the changes in the local and global tertiary structure of proteins upon mutations. Intrinsic fluorescence emission spectra were recorded between 300 and $400 \mathrm{~nm}$ upon excitation at $295 \mathrm{~nm}$. 8-aniline-1-naphthalene1-sulfonate (ANS), is an external hydrophobic fluorophore whose emission was scanned from 400 to $600 \mathrm{~nm}$ after excitation at $350 \mathrm{~nm}$. In extrinsic fluorescence, the photoprotein concentration was $1 \mu \mathrm{M}$ and the final ANS concentration in the protein solutions was $30 \mathrm{mM}$. Fluorescence spectra were performed with the fluorescence spectrophotometer (PerkinElmer LS 45) with a $1 \mathrm{~cm}$ path-length quartz cuvette. Both excitation and emission slits were set to $10 \mathrm{~nm}$.

\subsection{Thermal denaturation experiments}

Heat-induced denaturation experiments of $\mathrm{Mn} 2$ and individual mutants were performed by measuring the protein absorbance at $280 \mathrm{~nm}$ upon heating of protein solution in the temperature range of $4-90{ }^{\circ} \mathrm{C}$ using the UV/V is spectrophotometer (Jasco V730) equipped with the programmable Peltier. The experiments were performed in a $50 \mathrm{mM}$ dialysis buffer at $\mathrm{pH} 9$ with temperature changes of $5^{\circ} \mathrm{C}$ per minute. Protein concentration was $0.2 \mu \mathrm{M}$. The resulting sigmoid curve was plotted using KaleidaGraph software and analyzed using the relevant equations as will provided in the section "Results and discussion" [37].

\section{Result and discussion}

\subsection{Bioinformatics and designing of mutations}

The sequence of Mn2 from Mnemiopsis leidyi was aligned with the non-redundant similar protein sequences using the BLAST program. It was found that Mnemiopsis leidyi has different variants of photoproteins as well as Mn2. We also selected other photoproteins from the output results of BLAST program as summarized in Supplementary data 1.

The design of mutations for the Mn2 for current study was guided by examining the multiple sequence alignment of photoprotein sequences. Figure 1 shows the multiple sequence alignment between the photoprotein sequences.

According to MSA data of Fig. 1, positions 52 and 144 in Mn2 are occupied by Methionine and Valine, respectively. 


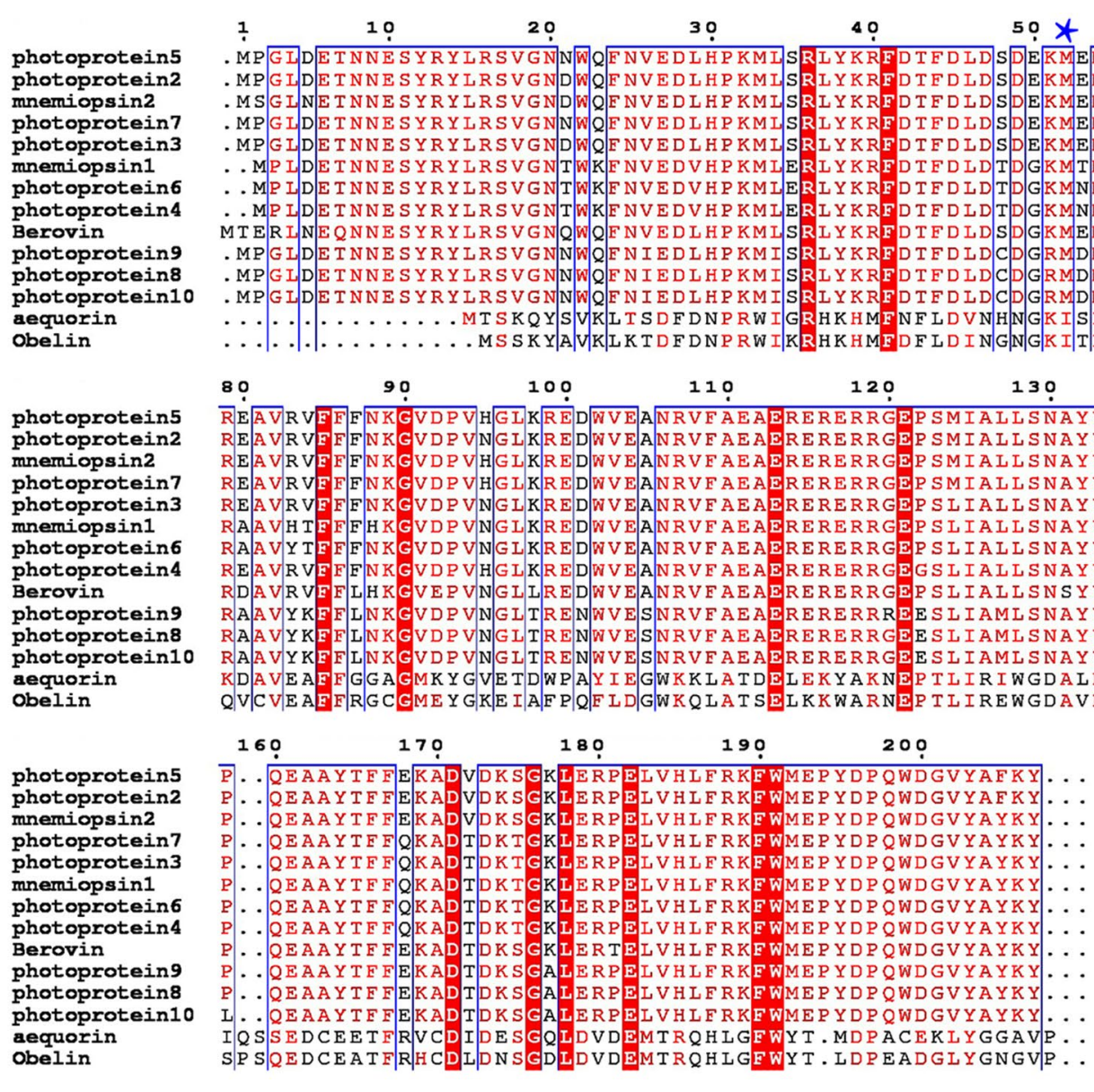

Fig. 1 Multiple sequence alignment between the photoprotein sequences. The sequences were aligned by the ClustalW program, and the output was used as input for the ESPript program to depict the final MSA file. The positions of the mutations were shown by "*”

However, exact examining of the conservation lines in these positions shows that Isoleucine is also found in other known photoproteins including Aequorin and Obelin that have higher activity relative to Mn2. Hence, regarding the advantages of aequorin compared to $\mathrm{Mn} 2$ in light emission and lower regeneration time, M52I and V144I mutations were designed in Mn2. To find the position of Met52 and Val144 in the context of the tertiary structure of Mn2, its three-dimensional structure was modelled with the MODELLER program (Fig. 2a and b).

As shown in panel a of Fig. 2, both positions are located in the loop structures adjacent to calcium ion. Accordingly, it is predicted that they may have important roles in the activity of the photoprotein. The structure of mutants including M52I and V144I were also constructed by the MODELLER program. Structure alignment of the WT and mutants are provided in Fig. $2 \mathrm{c}$ showing that the mutations do not change the overall structure of protein. According to Fig. $2 d$ all structures have the negative values of Z-Dope score, indicating that they have acceptable level of the structural quality for structure analysis.

\subsection{Expression and purification of photoproteins}

The purity of photoprotein variants after protein expression and purification was determined by the SDS-PAGE analysis. Figure 3 shows the results of SDS-PAGE analysis. Observing single bands near $27 \mathrm{kDa}$ for individual proteins demonstrates that the proteins have adequate purity for the activity measurements and structural studies.

\subsection{Activity measurement}

Decay rate along with initial luminescence activity are two physical parameters of light emission in the activity of photoproteins. Regarding the fact that the activity of aequorin as a member of Cnidarian family is more than that of $\mathrm{Mn} 2$ from Ctenophores, and assuming the significant role of the EF-hand I and III in the in the bioluminescence activity, we have expected that the mutations in the loops of Mn2 in accord with the corresponding positions of aequorin may leads to positive effect in the activity of mutants. However, activity measurements of the recombinant photoproteins 

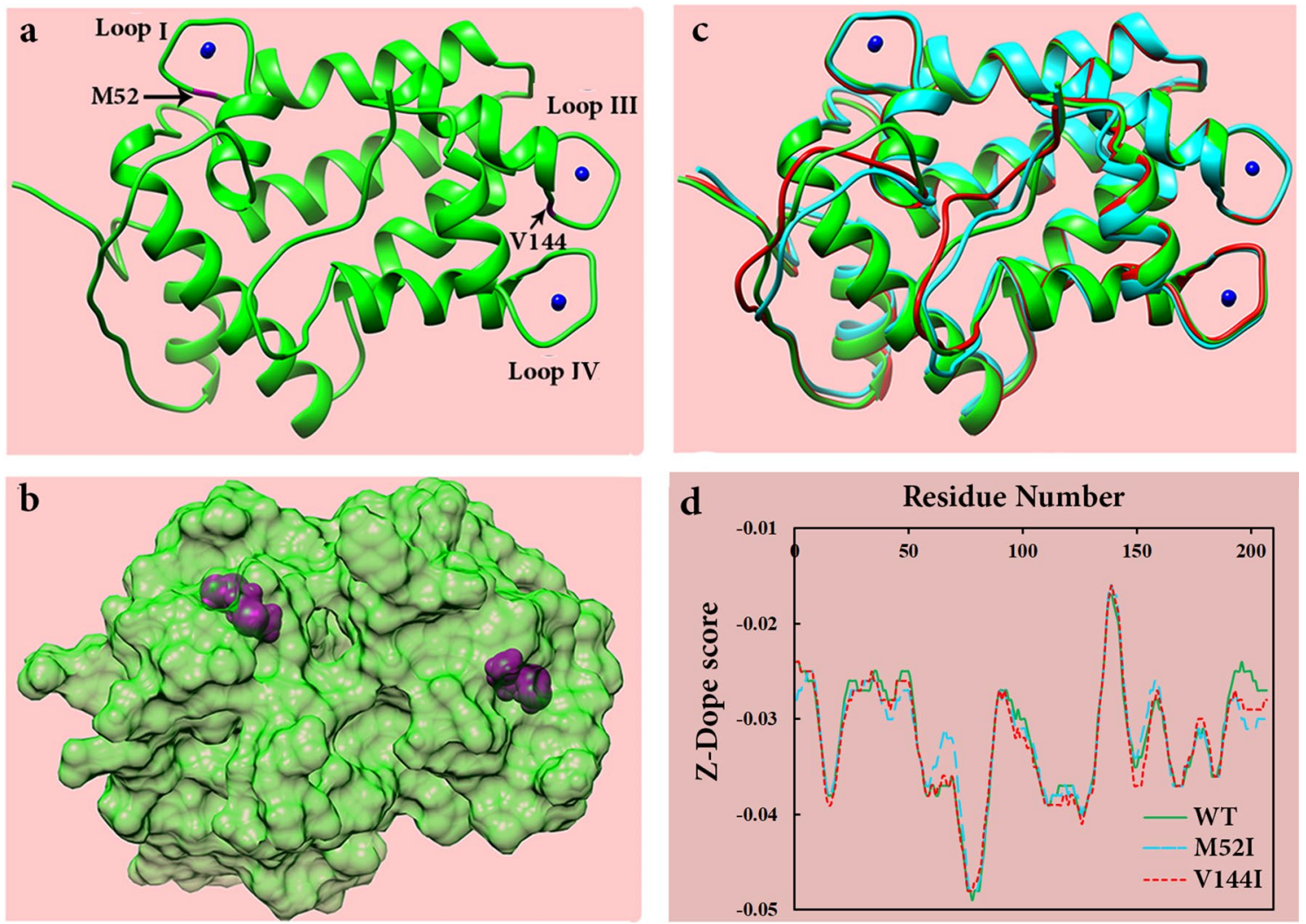

Fig. 2 Structural features of the WT Mn2 and its mutants. a Ribbon diagram of the three-dimensional structure of the WT Mn2 showing the positions of the mutations on loops I and III. b Surface representation of the WT Mn2 and the position of the surface-exposed residues as the site of mutations. c Superposition of the modelled

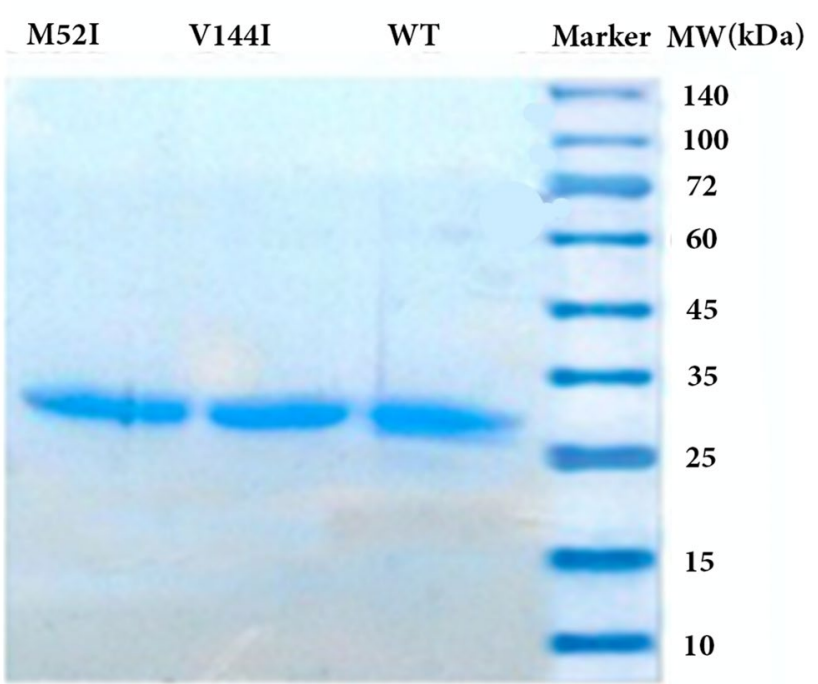

Fig. 3 SDS-PAGE analysis. SDS-PAGE gel was performed for characterization of the purity and yield of protein expression process. Numbers in the right represent the molecular weight of band positions in $\mathrm{kDa}$ structure of the WT and mutants of the Mn2 (d) Z-Dope score of the selected models. Depiction of the structures was performed by the UCSF Chimera program. For the clarity, substrate in the core structure of photoproteins are not shown

Table 1 Activity parameters for the WT photoprotein and its mutants

\begin{tabular}{lll}
\hline Photoprotein variants & Relative activity $(\%)$ & $\lambda_{\max }(\mathrm{nm})$ \\
\hline WT & 100 & 480 \\
M52I & 72 & 470 \\
V144I & 26 & 505 \\
\hline
\end{tabular}

The activity was reported as the percentage of the original activity of the WT photoprotein as the reference. $\lambda_{\max }$ is the recorded characteristic maximum wavelength

upon activation with the coelenterazine as the substrate indicated that the M52I and V144I mutants have only about $72 \%$ and $26 \%$ of the original activity of the WT protein, respectively (Table 1). It is also revealed that characteristic maximum wavelength of luminescence emission is changed upon mutation, where V1444I variant shows $25 \mathrm{~nm}$ red shift, and that for M52I is only $10 \mathrm{~nm}$ toward blue light as compared with the WT photoprotein. Unlike prediction, both mutants have reduced initial emission of bioluminescence than the WT Mn2. The decrease in the initial intensity of bioluminescence in mutants can be justified by considering 
the modification in the interaction of the core structure of the recombinant photoproteins upon mutation toward the instability of the functional complex. In statistical thermodynamics, it could be explained by reduction in the number of "pre-charged" functional complexes that results in the reduction in the number of emitted photons from the solution containing photoproteins.

Despite reduction in the initial intensity of the luminescence emission, observing $25 \mathrm{~nm}$ of red shift in the characteristic wavelengths for the V1444I variant compared with the WT photoprotein can be considered as an advantage for in vivo application of photoprotein for imaging purposes.

Decay rate of the bioluminescence activity as well as the calcium sensitivity curve for the WT Mn2 and its mutants are provided in Fig. 4, a and b. Tricoire et al. have reported that more sensitivity to calcium ions has led to an increase in decay rate [38]. Accordingly, it appears that decrease in the decay rate of V144I and M52I mutants compared with the WT Mn2 is originated from the reduction in the $\mathrm{Ca}^{+}$[2] sensitivity observed for mutants (Fig. 4a and b).

According to the intermediate-based model in explaining the bioluminescence activity of photoproteins and considering the formation of intermediate conformations in the light triggering process, the slow decay rate of V144I mutant could be attributed to decrease in the energy level of intermediate state and increasing its half-life. Ultimately, the photoprotein endure a longer time in activated state that results in decreasing the decay rate. According to calcium sensitivity curve of Fig. 4b, it appears that the binding affinity of $\mathrm{Ca}^{2+}$ to V144I variant is lower than that for WT and M52I mutant. Previous studies have shown that Mn2 has lower affinity to $\mathrm{Ca}^{+2}$ as compared with aequorin, and it is suitable for work in conditions of higher concentration of $\mathrm{Ca}^{+217}$. It is also reported that the $\mathrm{Ca}^{+2}$-triggered pattern of EF-hand loops I/III/IV to $\mathrm{Ca}^{+2}$ is different, and EF-hand loops III/IV play an important role in coordinate with $\mathrm{Ca}^{+2}$ ion than EF-hand loop I in Mn2 [39]. Hence, changing the pattern of $\mathrm{Ca}^{+2}$-triggering in V144I variant can be explained by modification of Loop III as a result of mutation.

\subsection{Spectroscopy}

To perceive how the M52I and V144I mutations have altered the secondary structural content of the Mn2, CD spectra in the far-UV region were measured. Figure 5a; illustrates the CD spectra of the WT and mutant photoproteins demonstrating that the whole shapes of CD spectra are almost similar for mutants and WT variants, but they have different intensities. In other words, observing a maximum peak below $200 \mathrm{~nm}$ and two minima at 208 and $222 \mathrm{~nm}$ shows a typical spectrum of $\alpha$-helical secondary structure. Decreasing the intensity of CD signals in mutants indicates that the peptide bonds involved in the helix structures are located in less
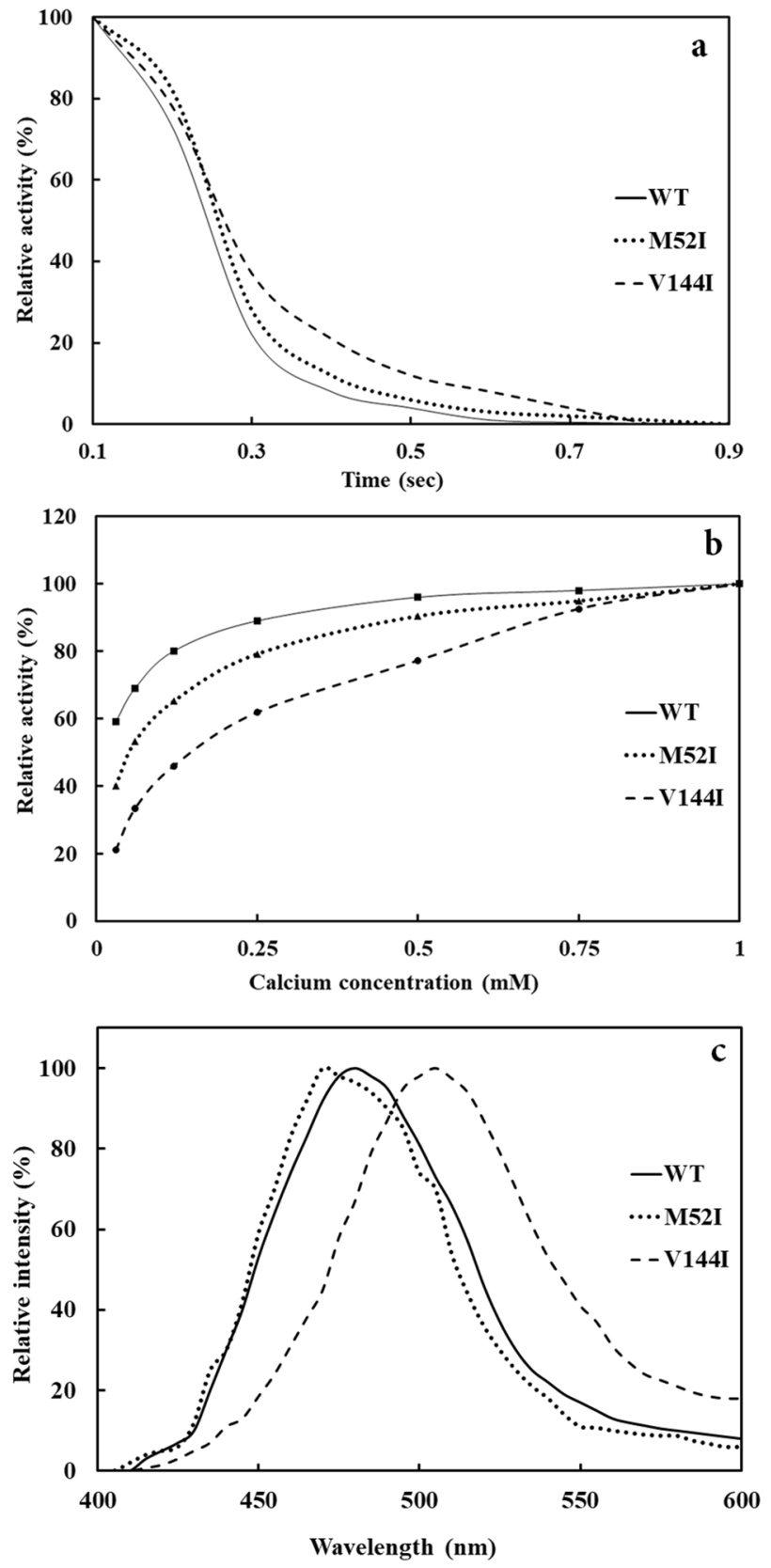

Fig. 4 a Decay rates, $\mathbf{b}$ calcium sensitivity and $\mathbf{c}$ emission spectra of the WT Mn2 and its mutants. The relative activity was reported as a percentage of the original activity of photoprotein variants. The bioluminescence signals were recorded at $0.1 \mathrm{~s}$ intervals for $10 \mathrm{~s}$

asymmetric structure. This means that the mutant proteins have less stabilizing interactions in their $\alpha$-helical structure, though their helical contents are the same as in WT protein.

To obtain local structural information around Trp residues, intrinsic fluorescence emission of photoprotein variants was recorded upon excitation at $295 \mathrm{~nm}$. Since, Trp residues as fluorophores have disparate levels of accessibility to the polar environment. So the ultimate fluorescence emission was 

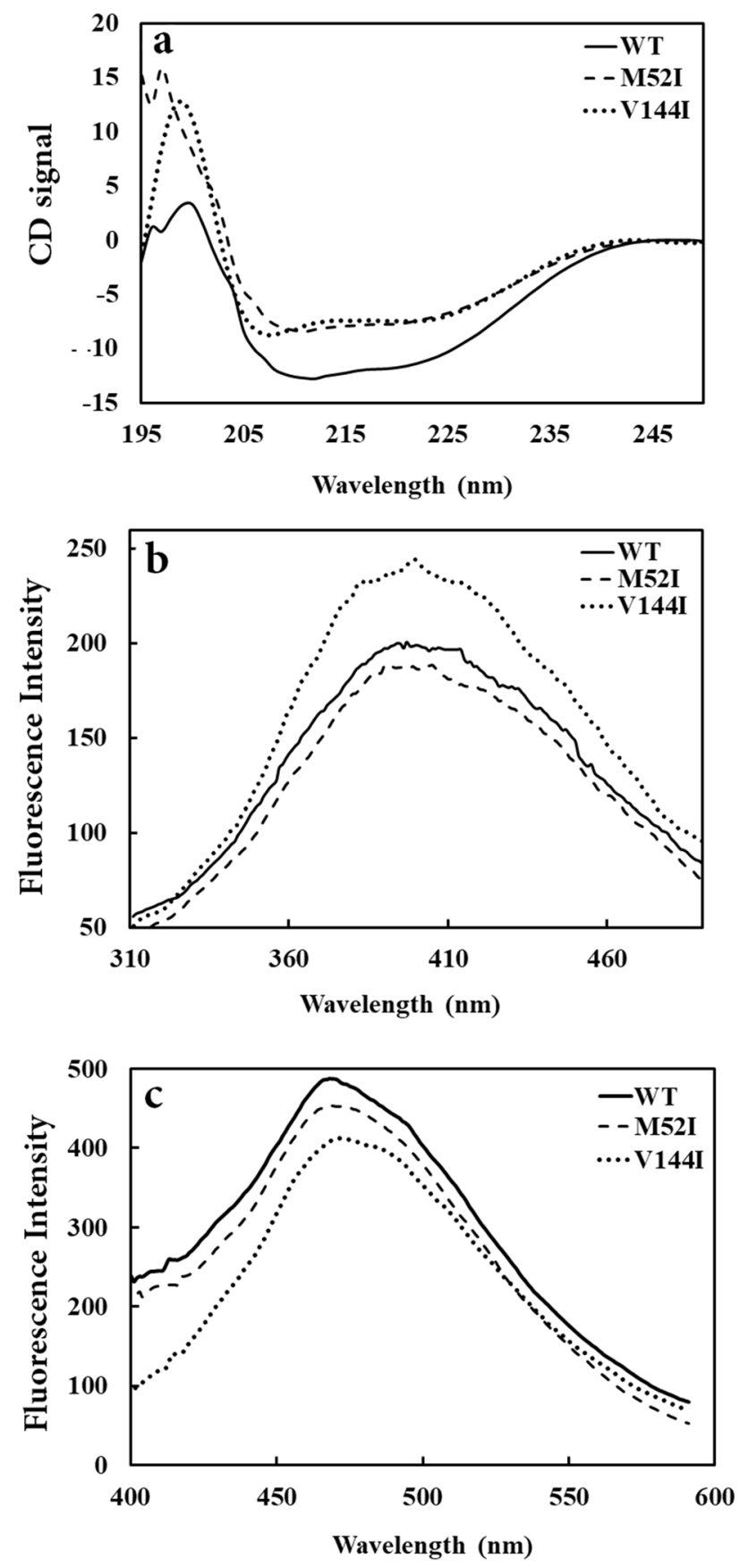

Fig. 5 Spectroscopy measurement. a Far-UV CD spectra of the WT $\mathrm{Mn} 2$ and its mutants. b Intrinsic fluorescence spectra of photoprotein variants. c ANS-based fluorescence of the WT and mutant photoproteins

included different contributions of Trp residues. Mn2 consists of 5 Trp residues and it was demonstrated that intrinsic fluorescence of $\mathrm{Mn} 2$ involves internal quenching [40]. Internal quenchers have absorbed the fluorescence emission of those Trp residues that are located near to them. Due to the intrinsic fluorescence data Fig. 5b; the fluorescence intensity of the V144I mutant increases as compared with the WT and M52I variants. This observation could be attributed to the structural rearrangements that caused the fluorophores to place far from the quenchers. Hence, the intrinsic fluorescence intensity increases upon the elimination of the quenching effect. To obtain global information about the tertiary structure of photoproteins, ANS fluorescence experiments were also performed. According to Fig. 4c, ANS fluorescence intensity in V144I mutant is reduced as compared with the WT and M52I variants. This observation is originated from the more compactness of the tertiary structure of this mutant which in turn caused the hydrophobic patches come into the internal regions of photoprotein. This leads to the reducing the number of binding sites for ANS molecules. A slightly red shift in the characteristic wavelength of the ANS fluorescence of V144I demonstrates that the strength of the ANS binding to the surface of protein decreases.

\subsection{Thermal denaturation experiments}

Structural alterations upon heating of WT and mutants of Mn2 were monitored by recording the absorbance of the protein solutions at $280 \mathrm{~nm}$. A general look at the curves shows that they have similar behavior. However, to obtain the quantitative insight on the thermal denaturation process, the sigmoidlike thermal denaturation curves were analyzed by a nonlinear least-square fit assuming two-state behavior according to Eq. 1 [37]:

$y=\frac{\left\{\left(\left(\alpha_{\mathrm{N}}+\beta_{\mathrm{N}} T\right)+\left(\alpha_{\mathrm{D}}+\beta_{\mathrm{D}} T\right) \times \exp \left(\left(\frac{\Delta H}{R}\right)\left(\frac{1}{T_{\mathrm{m}}}-\frac{1}{T}\right)\right)\right)\right.}{\left.\left(1+\exp \left(\left(\frac{\Delta H}{R}\right)\left(\frac{1}{T_{\mathrm{m}}}-\frac{1}{T}\right)\right)\right)\right\}}$,

where $\alpha_{\mathrm{N}}$ and $\beta_{\mathrm{N}}$ are the pre-transition baseline and its slope, respectively. $\alpha_{\mathrm{D}}$ and $\beta_{\mathrm{D}}$ are the post-transition baseline and its slope, respectively, $y$ is the measured spectroscopic signal, $\Delta H_{\mathrm{vh}}$ is the enthalpy of the unfolding transition, $T_{\mathrm{m}}$ is midpoint of the transition. In statistical view, it is a temperature in which half of the protein population are denatured. Also it can be considered as the temperature in which the $50 \mathrm{~g} \%$ of the stabilizing interactions of a protein molecule are lost.

$T$ is the temperature in Kelvin and $\mathrm{R}$ is the universal gas constant.

Upon fitting of the experimental data of Fig. 6 into Eq. 1 two main thermodynamic parameters of the unfolding process are obtained.

In the $T_{\mathrm{m}}$ of the structural transition, the change in Gibbs free energy (Eq. 2) is zero, accordingly:

$\Delta G=\Delta H-T \Delta S$,

$\Delta H-T_{\mathrm{m}} \Delta S=0$, 

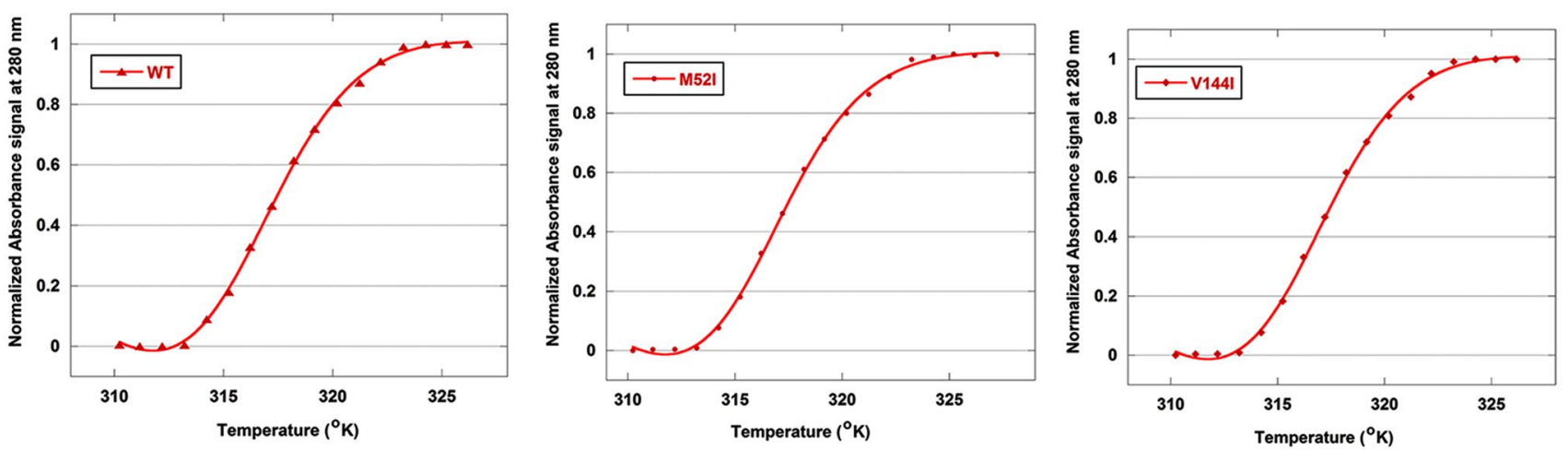

Fig. 6 Thermal denaturation curves of photoprotein variants. The absorbance at $280 \mathrm{~nm}$ was monitored by UV/Vis spectroscopy equipped with the Peltier. Data points are experimentally recorded signal, and the continuous lines are the best fitting of the experimental data into Eq. 1

Table 2 Thermodynamic parameters of WT Mn2 and its mutant in heat-induced denaturation experiments

\begin{tabular}{lccl}
\hline Protein variant & $\Delta \mathrm{H}(\mathrm{kcal} / \mathrm{mole})$ & $\mathrm{T}_{\mathrm{m}}$ (Kelvin) & $\begin{array}{l}\Delta \mathrm{S}(\mathrm{Cal} / \\
\text { mole Kel- } \\
\text { vin })\end{array}$ \\
\hline WT & $102 \pm 0.2$ & $316.7 \pm 0.1$ & $322 \pm 0.6$ \\
M52I & $93 \pm 0.3$ & $316.3 \pm 0.1$ & $294 \pm 0.1$ \\
V144I & $91 \pm 0.2$ & $316.3 \pm 0.1$ & $287 \pm 0.1$ \\
\hline
\end{tabular}

Data are reported as the mean \pm standard deviation of repeated experiments

$\Delta H=T_{\mathrm{m}} \Delta S$,

$\Delta S=\frac{\Delta H}{T_{\mathrm{m}}}$

Therefore, the values of the entropy change for the unfolding process can be calculated using Eq. 5. The thermal unfolding parameters are provided in Table 2.

The higher value of enthalpy change for the WT photoprotein indicates that structural change of this variant are accompanied by more energy exchange. In other words, it appears that mutation leads to energetic expenditure due to unfavorable interactions of the surfaced-exposed Isoleucine residues at positions 52 and 144, and decreasing the stabilizing interaction for the whole structure of the photoprotein. In spite of variation in the value of the enthalpy change, the protein variants shows the same values of $T_{\mathrm{m}}$ for the unfolding process, indicating that the structural transition for all protein variants occurred in a similar manner. To compare the thermal stability of protein variants considering enthalpy and entropy change, the Gibbs free energy change of the unfolding process at different temperatures can be calculated using data of Table 2

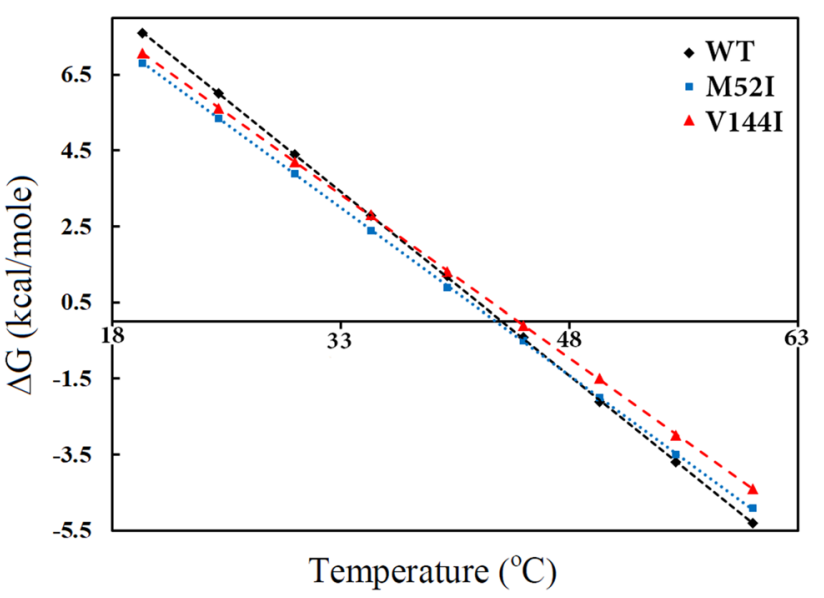

Fig. 7 Gibbs free energy change of the heat-induced denaturation process calculated for different temperatures by Eq. 2 using the numerical values of $\Delta H$ and $\Delta S$ as provided in Table 2

and Eq. 5. The Gibbs free energy change of the unfolding process at a range of the temperature is shown in Fig. 7.

According to data of Fig. 7, all protein variants shows a linear dependence to the temperature change in which the structural transition toward unfolding linearly increases with increasing temperature. However, the slope of the linear dependence is slightly different for protein variants. At moderate temperatures the WT protein is more stable than mutants, while in higher temperature the WT gains the minimum stability as compared with the mutants.

\section{Conclusion}

According to structural studies, positions 52 and 144 are located in the EF-hands loops I and III of Mn2, respectively. Multiple sequence alignment of Mn2 with similar photoproteins indicates that these positions have some degrees 
of variability under the evolutionary driving force, and the mutations on positions 52 and 144 are tolerable. Activity measurements on the WT and mutants of Mn2 showed that M52I and V144I have lower initial intensity as well as slower decay rate in comparison with the WT photoprotein. However, observing $25 \mathrm{~nm}$ of red shift in the characteristic wavelengths of the V144I variant, make this mutant prone for more studies to increase the stability and initial intensity of the bioluminescence activity for practical purposes.

Supplementary Information The online version contains supplementary material available at https://doi.org/10.1007/s43630-022-00191-6.

Acknowledgements We acknowledge the research council of the University of Zanjan.

\section{Declarations}

Statements and declarations Partial financial support was received from the research council of the University of Zanjan. The authors declare they have no financial interests, and they have no relevant financial or non-financial interests to disclose.

\section{References}

1. Haddock, S. H. D., Moline, M. A., \& Case, J. F. (2010). Bioluminescence in the sea. Annual Review of Marine Science, 2, 443-493.

2. Prendergast, F. G. (2000). Bioluminescence illuminated. Nature, 405, 291-292.

3. Kaskova, Z. M., Tsarkova, A. S., \& Yampolsky, I. V. (2016). 1001 lights: Luciferins, luciferases, their mechanisms of action and applications in chemical analysis, biology and medicine. Chemical Society Reviews, 45, 6048-6077.

4. Rees, J. F., \& Thompson, E. M. (1994). Photophores: The analysis of bioluminescent systems. Biochemistry Molecular Biology of Fishes, 3, 215-229.

5. Burakova, L. P., \& Vysotski, E. S. (2019). Recombinant Ca2+regulated photoproteins of ctenophores: Current knowledge and application prospects. Applied Microbiology and Biotechnology, 103, 5929-5946.

6. Deng, L., Markova, S. V., Vysotski, E. S., Liu, Z. J., Lee, J., Rose, J., \& Wang, B. C. (2004). Crystal structure of a Ca2+-discharged photoprotein. Implications for mechanisms of the calcium trigger and bioluminescence. Journal of Biological Chemistry, 279, 33647-33652.

7. Dikici, E., Qu, X., Rowe, L., Millner, L., Logue, C., Deo, S. K., Ensor, M., \& Daunert, S. (2009). Aequorin variants with improved bioluminescence properties. Protein Engineering, Design \& Selection, 22, 243-248.

8. Blinks, J. R. (1990). Use of photoproteins as intracellular calcium indicators. Environment Health Perspectives, 84, 75-81.

9. Stepanyuk, G. A., Liu, Z. J., Burakova, L. P., Lee, J., Rose, J., Vysotski, E. S., \& Wang, B. C. (2013). Spatial structure of the novel light-sensitive photoprotein berovin from the ctenophore Beroe abyssicola in the $\mathrm{Ca} 2+$-loaded apoprotein conformation state. Biochimica et Biophysica Acta (BBA) Proteins and Proteomics, 1834, 2139-2146.

10. Burakova, L. P., Natashin, P. V., Malikova, N. P., Niu, F., Pu, M., Vysotski, E. S., \& Liu, Z. J. (2016). All Ca2+-binding loops of light-sensitive ctenophore photoprotein berovin bind magnesium ions: The spatial structure of $\mathrm{Mg} 2$ +-loaded apo-berovin. Journal of Photochemistry and Photobiology, B: Biology, 154, $57-66$.

11. Head, J. F., Inouye, S., Teranishi, K., \& Shimomura, O. (2000). The crystal structure of the photoprotein aequorin at $2.3 \mathrm{~A}$ resolution. Nature, 405, 372-376.

12. Kawasaki, H., Nakayama, S., \& Kretsinger, R. H. (1998). Classification and evolution of EF-hand proteins. BioMetals, 11, 277-295.

13. Nelson, M. R., \& Chazin, W. J. (1998). Structures of EF-hand $\mathrm{Ca} 2+$-binding proteins: Diversity in the organization, packing and response to $\mathrm{Ca} 2+$ binding. BioMetals, 11, 297-318.

14. Grabarek, Z. (2006). Structural basis for diversity of the EF-hand calcium-binding proteins. Journal of Molecular Biology, 359, 509-525.

15. Tricoire, L., Tsuzuki, K., Courjean, O., Gibelin, N., Bourout, G., Rossier, J., \& Lambolez, B. (2006). Calcium dependence of aequorin bioluminescence dissected by random mutagenesis. Proceedings of the National Academy of Science U. S. A., 103, 9500-9505.

16. Brini, M. (2008). Calcium-sensitive photoproteins. Methods, 46, 160-166.

17. Ward, W. W., \& Seliger, H. H. (1974). Properties of mnemiopsin and berovin, calcium-activated photoproteins from the ctenophores mnemiopsis species and beroe ovata. Biochemistry, 13, $1500-1510$.

18. Mao, D., Wu, W., Ji, S., Chen, C., Hu, F., Kong, D., Ding, D., \& Liu, B. (2017). Chemiluminescence-guided cancer therapy using a chemiexcited photosensitizer. Chem, 3, 991-1007.

19. Frank, L. A., Borisova, V. V., Markova, S. V., Malikova, N. P., Stepanyuk, G. A., \& Vysotski, E. S. (2008). Violet and greenish photoprotein obelin mutants for reporter applications in dual-color assay. Analytical and Bioanalytical Chemistry, 391, 2223-2225.

20. Rowe, L., Ensor, M., Mehl, R., \& Daunert, S. (2010). Modulating the bioluminescence emission of photoproteins by in vivo sitedirected incorporation of non-natural amino acids. ACS Chemical Biology, 5, 455-460.

21. Rowe, L., Dikici, E., \& Daunert, S. (2009). Engineering bioluminescent proteins: Expanding their analytical potential. Analytical Chemistry, 81, 8662-8668.

22. Aghamaali, M. R., Jafarian, V., Sariri, R., Molakarimi, M., Rasti, B., Taghdir, M., Sajedi, R. H., \& Hosseinkhani, S. (2011). Cloning, sequencing, expression and structural investigation of mnemiopsin from mnemiopsis leidyi: an attempt toward understanding Ca 2+- regulated photoproteins. Protein Journal, 30, 566-574.

23. Ward, W. W., \& Seliger, H. H. (1974). Extraction and purification of calcium-activated photoproteins from the ctenophores mnemiopsis species and beroe ovata. Biochemistry, 13, 1491-1499.

24. Ghanbarlou, R. M., Shirdel, S. A., Jafarian, V., \& Khalifeh, K. (2018). Molecular mechanisms governing the evolutionary conservation of Glycine in the 6th position of loops III and IV in photoprotein mnemiopsin 2. Journal of Photochemistry Photobiology B Biology, 187, 18-24.

25. Altschul, S. F., Gish, W., Miller, W., Myers, E. W., \& Lipman, D. J. (1990). Basic local alignment search tool. Journal of Molecular Biology, 215, 403-410.

26. Rice, P., Longden, L., \& Bleasby, A. (2000). EMBOSS: The European molecular biology open software suite. Trends in Genetics, $16,276-277$.

27. Higgins, D. G., Thompson, J. D., \& Gibson, T. J. (1996). Using CLUSTAL for multiple sequence alignments. Methods in Enzymology, 266, 383-402.

28. Gouet, P., Courcelle, E., Stuart, D. I., \& Métoz, F. (1999). ESPript: Analysis of multiple sequence alignments in PostScript. Bioinformatics, 15, 305-308. 
29. Fiser, A., \& Šali, A. (2003). MODELLER: generation and refinement of homology-based protein structure models. Methods in Enzymology, 374, 461-491.

30. Head, J. F., Inouye, S., Teranishi, K., \& Shimomura, O. (2000). The crystal structure of the photoprotein aequorin at $2.3 \AA$ resolution. Nature, 405, 372-376.

31. Shen, M.-Y., \& Sali, A. (2006). Statistical potential for assessment and prediction of protein structures. Protein Science, 15, 2507-2524.

32. Pettersen, E. F., Goddard, T. D., Huang, C. C., Couch, G. S., Greenblatt, D. M., Meng, E. C., \& Ferrin, T. E. (2004). UCSF Chimera- a visualization system for exploratory research and analysis. Journal of Computational Chemistry, 25, 1605-1612.

33. Jafarian, V., Sariri, R., Hosseinkhani, S., Aghamaali, M. R., Sajedi, R. H., Taghdir, M., \& Hassannia, S. (2011). A unique EFhand motif in mnemiopsin photoprotein from Mnemiopsis leidyi: Implication for its low calcium sensitivity. Biochemical and Biophysical Research Communications, 413, 164-170.

34. Bradford, M. M. (1976). A rapid and sensitive method for the quantitation of microgram quantities of protein utilizing the principle of protein-dye binding. Analytical Biochemistry, 72, $248-254$.

35. Inouye, S., \& Sahara-miura, Y. (2016). Expression and characterization of EF-hand I loop mutants of aequorin replaced with other loop sequences of $\mathrm{Ca} 2+-$ binding proteins: An approach to studying the EF-hand motif of proteins. Journal of Biochemistry, $160,59-68$.

36. Inouye, S., \& Sahara, Y. (2007). Expression, purification and characterization of a photoprotein, clytin, from Clytia gregarium. Protein Expression and Purification, 53, 384-389.

37. Shirdel, S. A., \& Khalifeh, K. (2019). Thermodynamics of protein folding: Methodology, data analysis and interpretation of data. European Biophysics Journal, 48, 305-316.

38. Calcium dependence of aequorin bioluminescence dissected by random mutagenesis, Herring PJ. Some Featur. Biolumin. Radiol. Thalass. sp. Mar. (1979). Biol. 53213-16.

39. Jafarian, V., Sajedi, R. H., Hosseinkhani, S., Sariri, R., Taghdir, M., Khalifeh, K., Vafa, M., \& Aghamaali, M. R. (2018). Structural and functional consequences of EF-hand I recovery in mnemiop$\sin$ 2. International Journal of Biological Macromolecules, 118, 2006-2013.

40. Vafa, M., Khalifeh, K., \& Jafarian, V. (2018). Negative net charge of EF-hand loop I can affect both calcium sensitivity and substrate binding pattern in mnemiopsin 2. Photochemical \& Photobiological Sciences, 17, 807-814. 